IMTEK

\title{
Polymers with Customizable Optical and Rheological Properties based on an Epoxy Acrylate based Host-Guest System
}

Uwe Gleißner, Jost Hobmaier, Thomas Hanemann

Laboratory for Materials Processing

Department of Microsystems Engineering - IMTEK

University of Freiburg

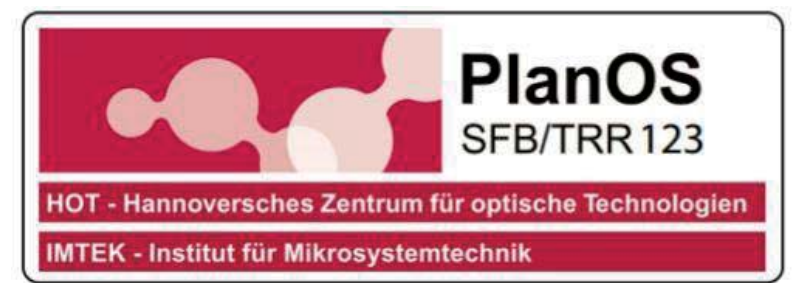




\section{Planar Optronic Systems}

\section{Collaborative Research Center}

- Polymer-based sensor network

- Large-area foils

- No electronic components

- Measurement of

> Temperature

$>$ Strain

- Sub-projects

Suitable materials

Construction of fiber optics

$>$ Light sources

Spectrometers / detectors

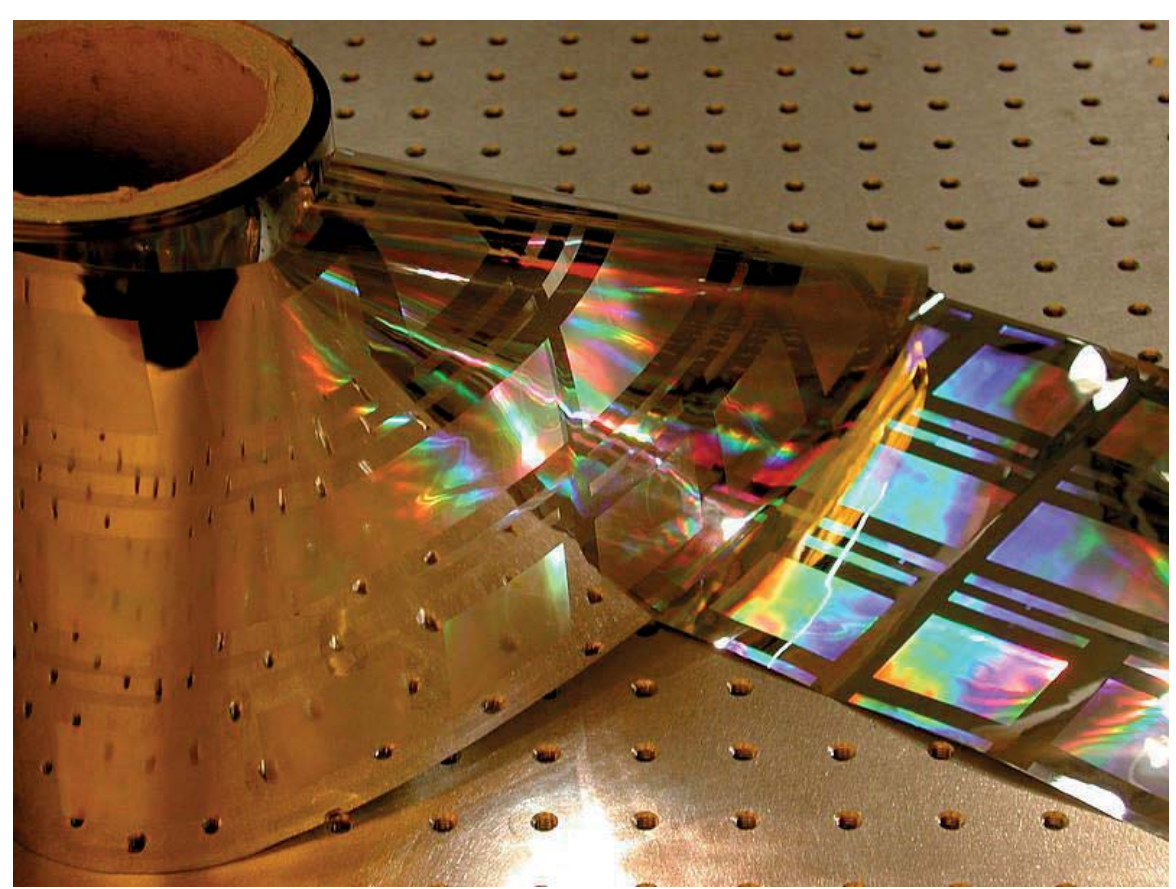

polymer foil

[http://www.planos.uni-hannover.de] 


\section{Planar Optronic Systems}

\section{Why polymers?}

- Modifiable to application

- Good processability

Hot embossing

$>\mathrm{NIL}$

Inkjet-printing

- Large-scale systems possible

- Thin layers = economic

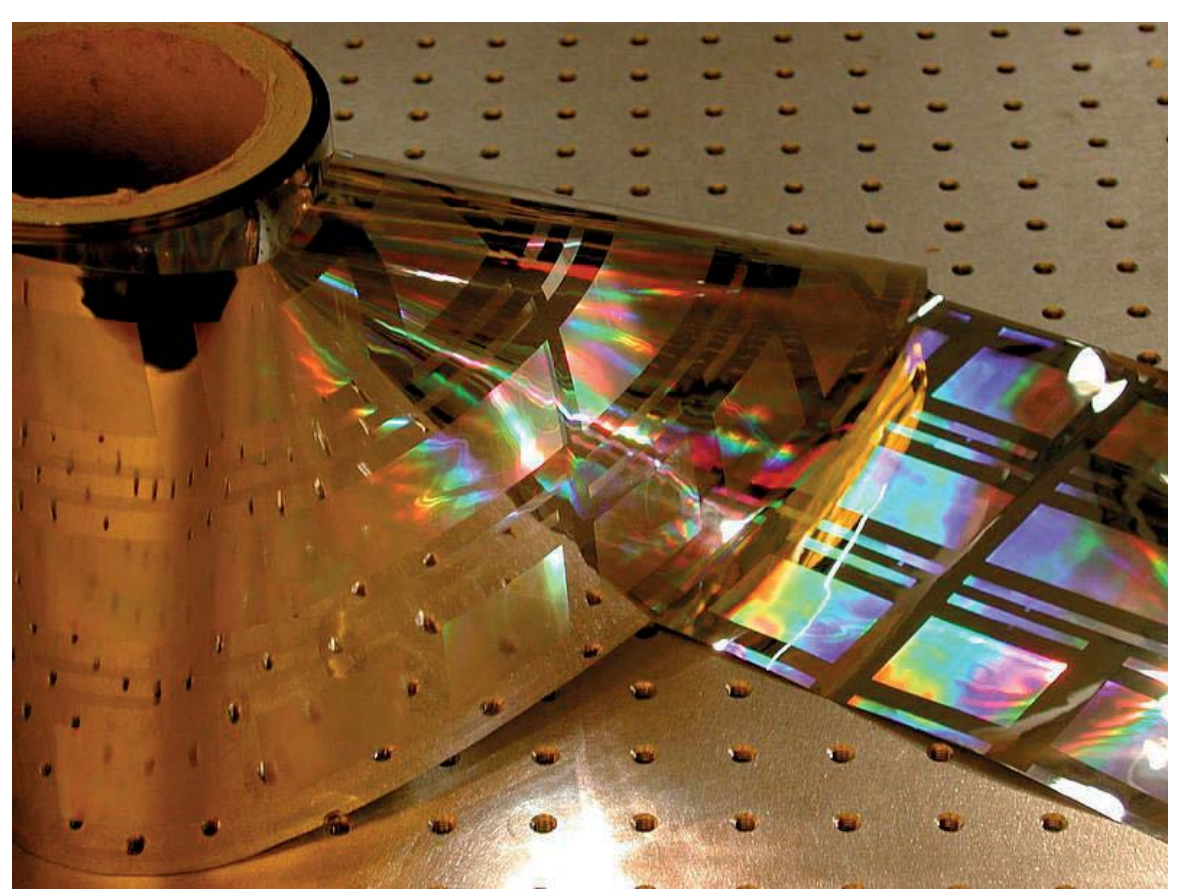

polymer foil

[http://www.planos.uni-hannover.de] 


\section{Planar Optronic Systems}

\section{Tailored polymers}

Adjusting viscosity

- Polymerization by UV-light

- Adjusting refractive indices

- Low optical damping

- Continuous operating temperature

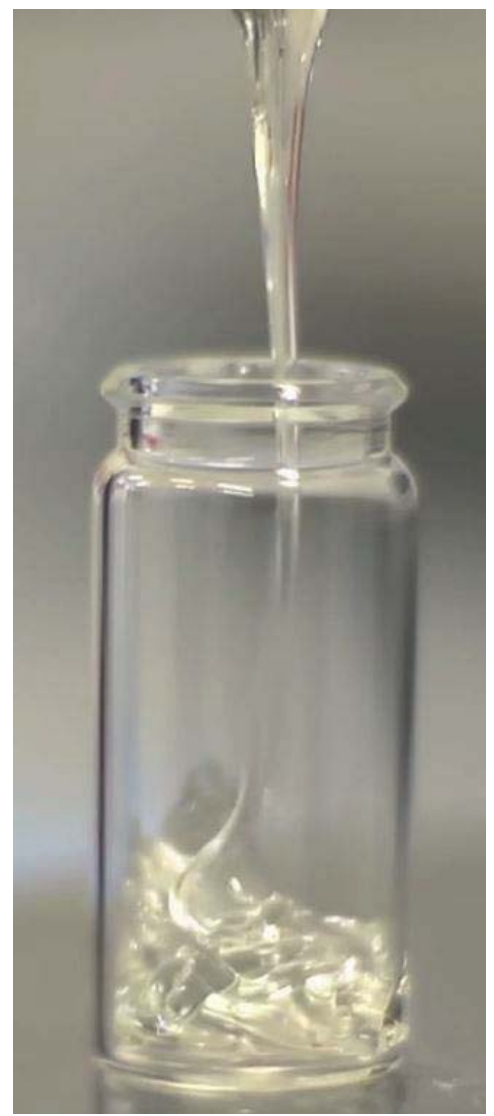




\section{Planar Optronic Systems}

\section{Tailoring viscosity}

Comonomer content

Different shaping / molding

processes

$>$ Inkjet printing

$$
\approx 10 \mathrm{mPa} \cdot \mathrm{s}\left(@ 70^{\circ} \mathrm{C}\right)
$$

$>$ Offset printing

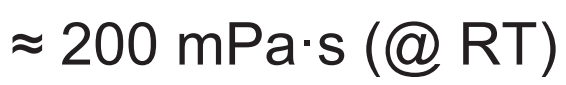

Spin coating

$$
\approx 100 \mathrm{mPa} \cdot \mathrm{s}-1000 \mathrm{mPa} \cdot \mathrm{s} \text { (@ RT) }
$$

Dimatix DMP 2831 [www.electronicdata.com]

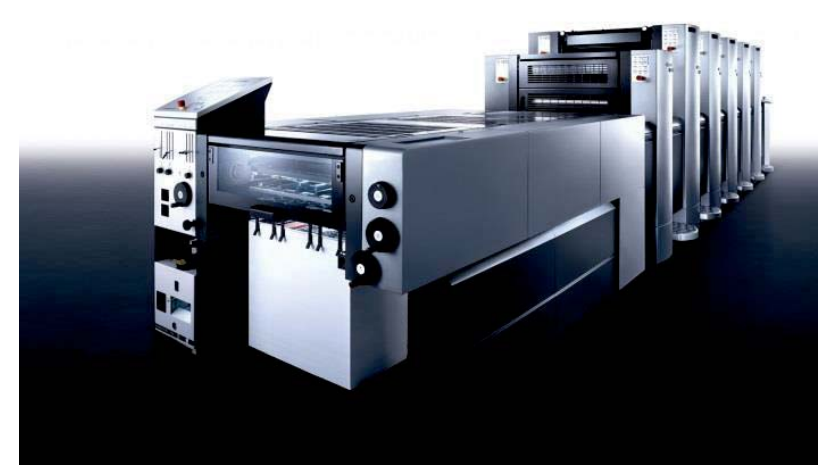

Heidelberg Speedmaster SM 52 [www.heidelberg.com] 


\section{Planar Optronic Systems}

\section{Adjustment of refractive indices}

Comonomer / dopant

- Waveguides

$>$ Core

Cladding

Coupling structures

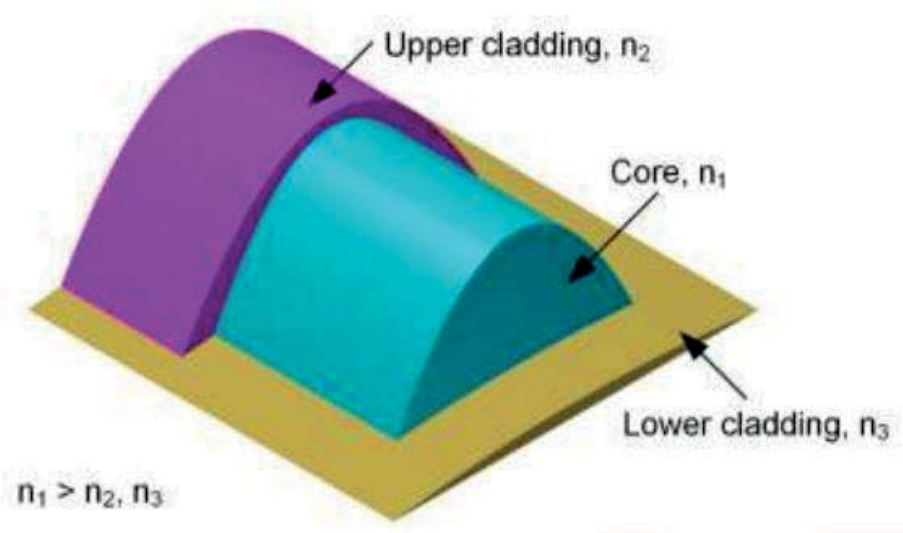

Computed 3D model of printed waveguide (Wolfer et al, Procedia Technology, 2013) 


\section{Planar Optronic Systems}

\section{Materials}

- Main monomer

- Epoxy Methacrylate 97-053 (RAHN)

- Comonomer

- Benzyl methacrylate

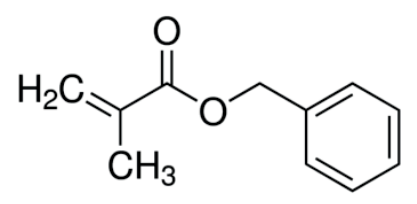

- Dopant

Phenanthrene

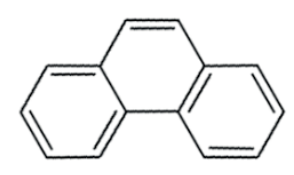

- UV initiator

$>$ Phosphine oxide

- Thermal initiator

$>$ Lauroyl peroxide

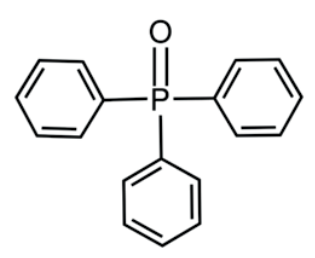




\section{Planar Optronic Systems}

\section{Radical polymerization}
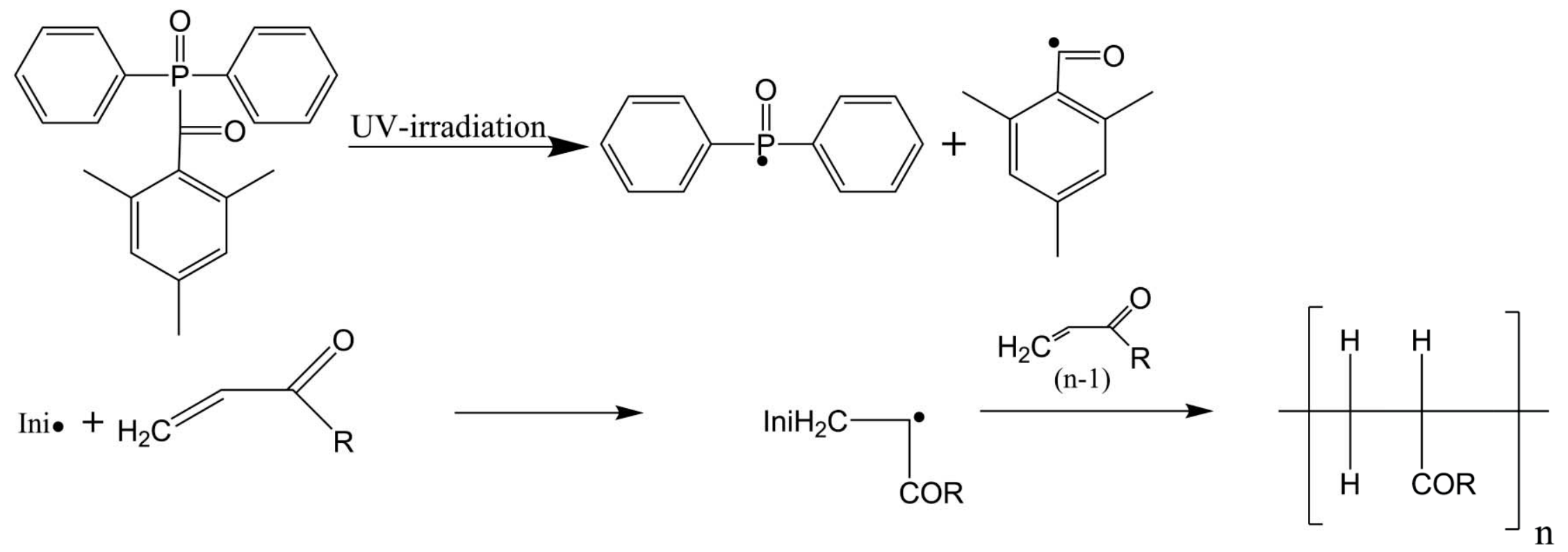


\section{Planar Optronic Systems}

\section{Mixture preparation}

- Materials are mixed

up to $30.000 \mathrm{rpm}$

$>$ ambient conditions

- Ultrasonic bath

- Viscosity measurement

Cone and plate rheometer
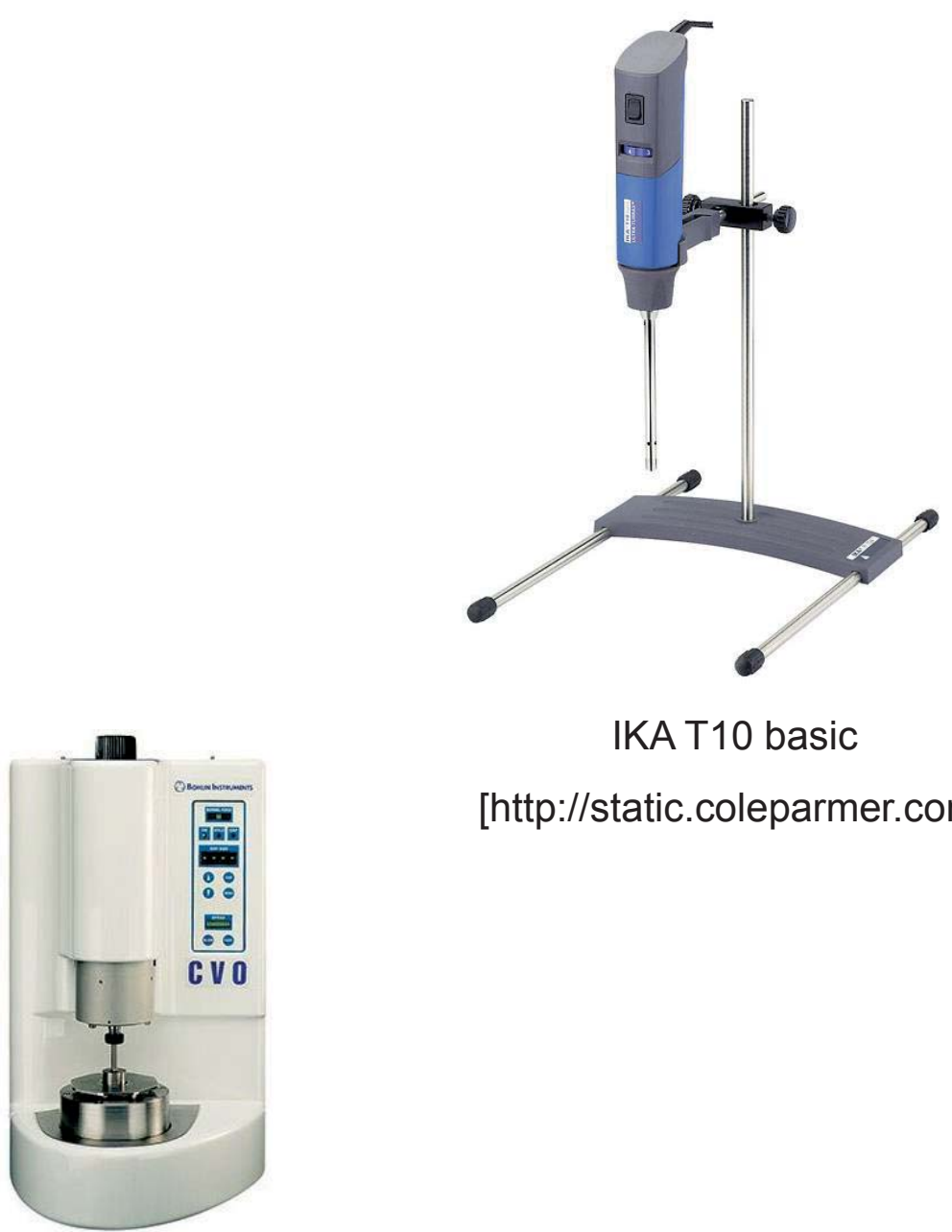

IKA T10 basic

[http://static.coleparmer.com]

Bohlin Rheometer CVO 50

[http://mb.uni-paderborn.de/] 


\section{Planar Optronic Systems}

\section{Sample preparation}

- For refractive index

Casting mold (silicon)

Glass plates

Fluorine ethylene propylene (FEP) foil

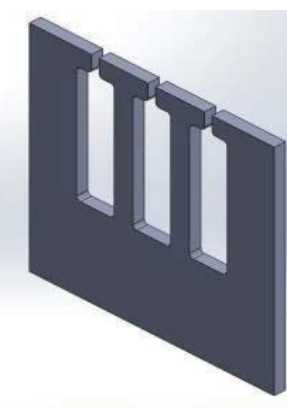

casting mold

- Oxygen inhibition 


\section{Planar Optronic Systems}

\section{Sample preparation}

- For refractive index

Casting mold (silicon)

Glass plates

Fluorine ethylene propylene (FEP) foil

- Oxygen inhibition

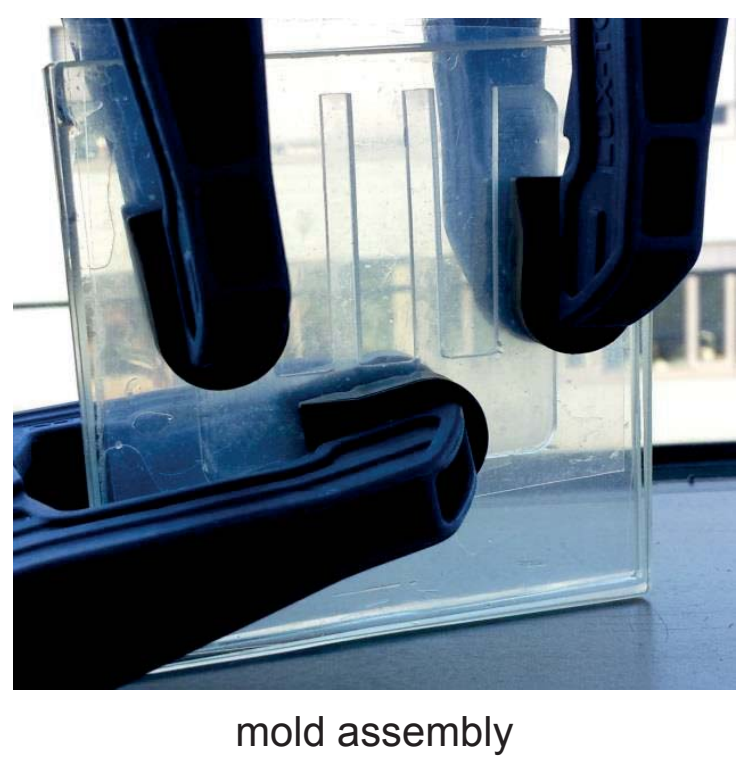




\section{Planar Optronic Systems}

\section{Sample preparation}

- For refractive index

$>$ Casting mold (silicon)

Glass plates

$>$ Fluorine ethylene propylene (FEP) foil

- Oxygen inhibition

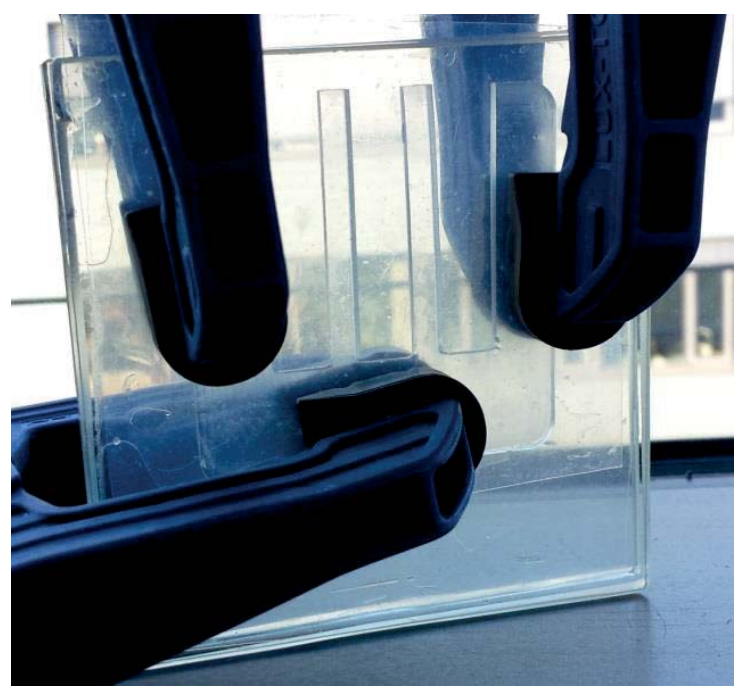

mold assembly

- Polymerization

Wavelength $405 \mathrm{~nm}$

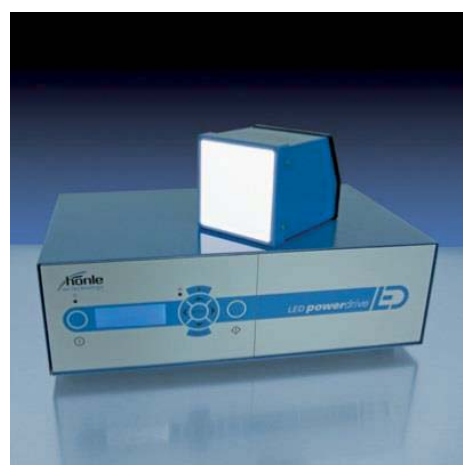

Hönle UV-Spot 100

[www.hoenle.de] 


\section{Planar Optronic Systems}

\section{Sample preparation}

- For refractive index

Casting mold (silicon)

$>$ Glass plates

Fluorine ethylene propylene (FEP) foil

- Oxygen inhibition

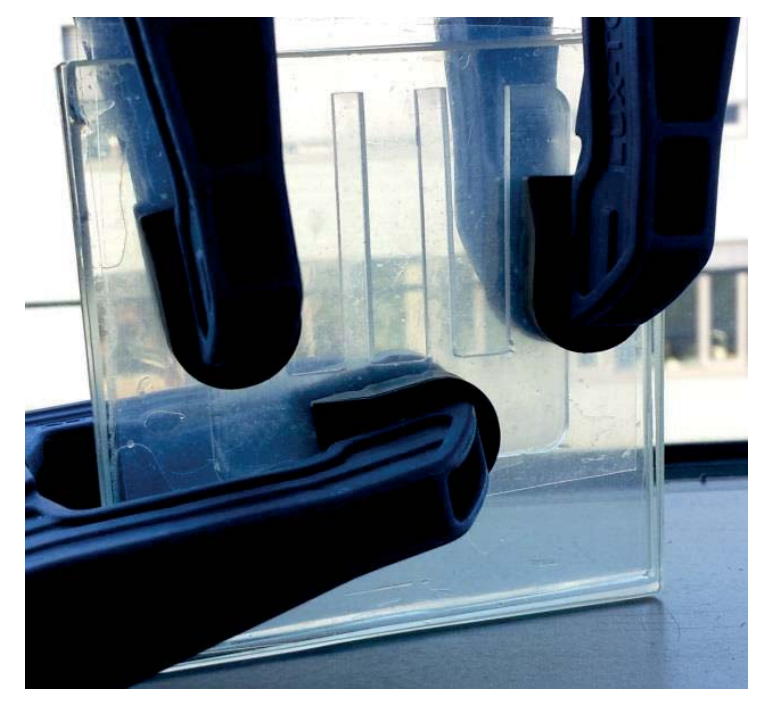

mold assembly

- Polymerization

Wavelength $405 \mathrm{~nm}$

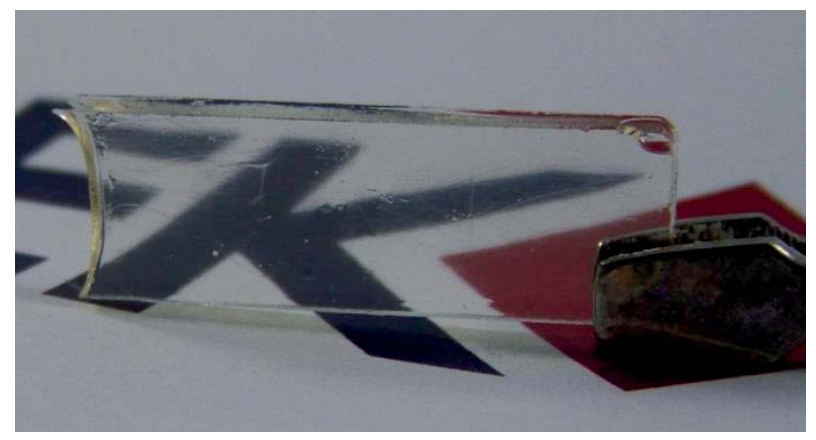

polymerized sample 


\section{Planar Optronic Systems}

\section{Sample characterization}

- Refractive indices

- Abbe-refractometer

$>$ Multi-wavelength

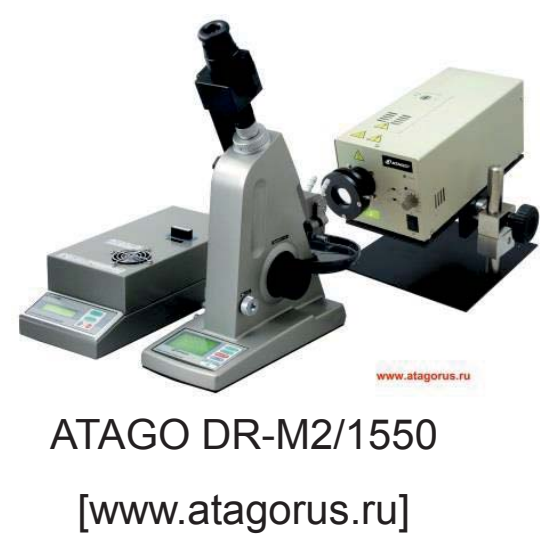

- Optical damping

UV-Vis spectroscopy

- Differential scanning calorimetry (DSC)

$>$ Glass transition temperature

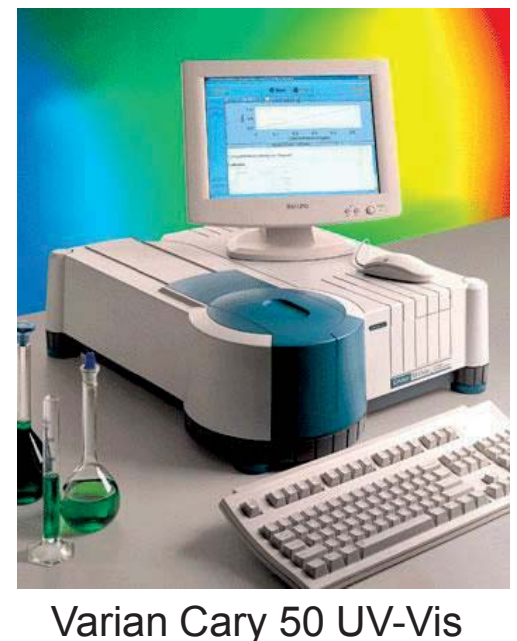

Varian Cary 50 UV-Vis

[www.speciation.net]

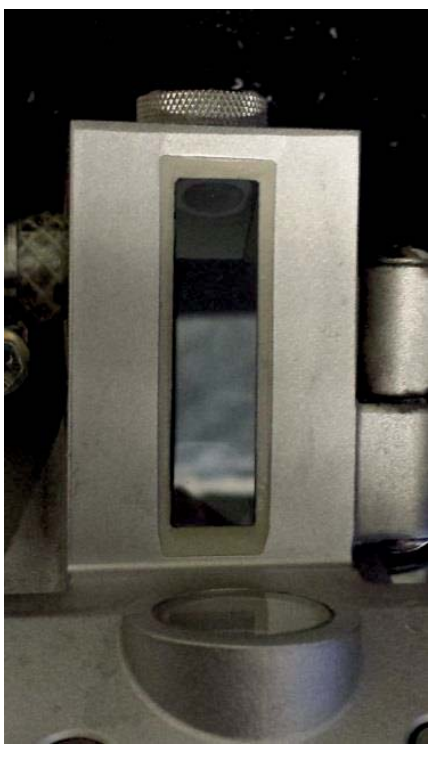

refractometer surface 


\section{Planar Optronic Systems}

\section{Viscosity of Epoxy Methacrylate 97-053 + BMA}

Temperature dependency

Newtonian behavior

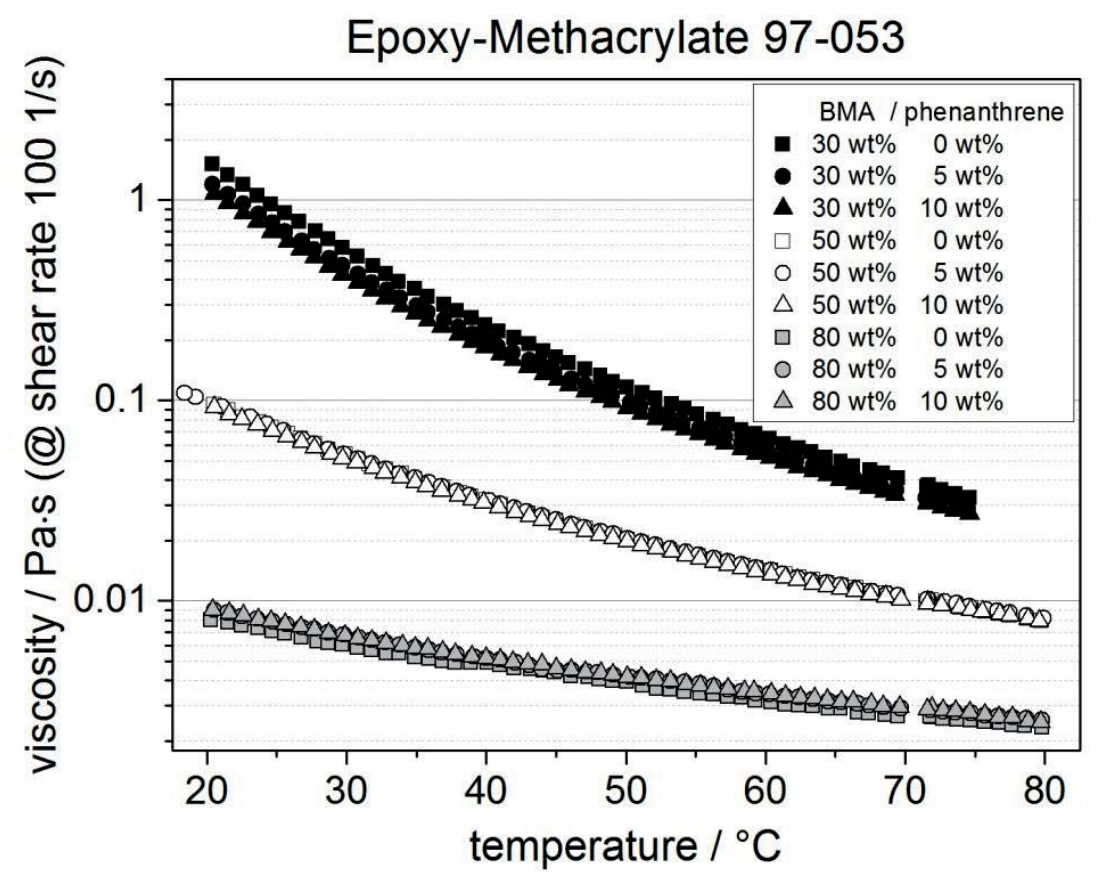

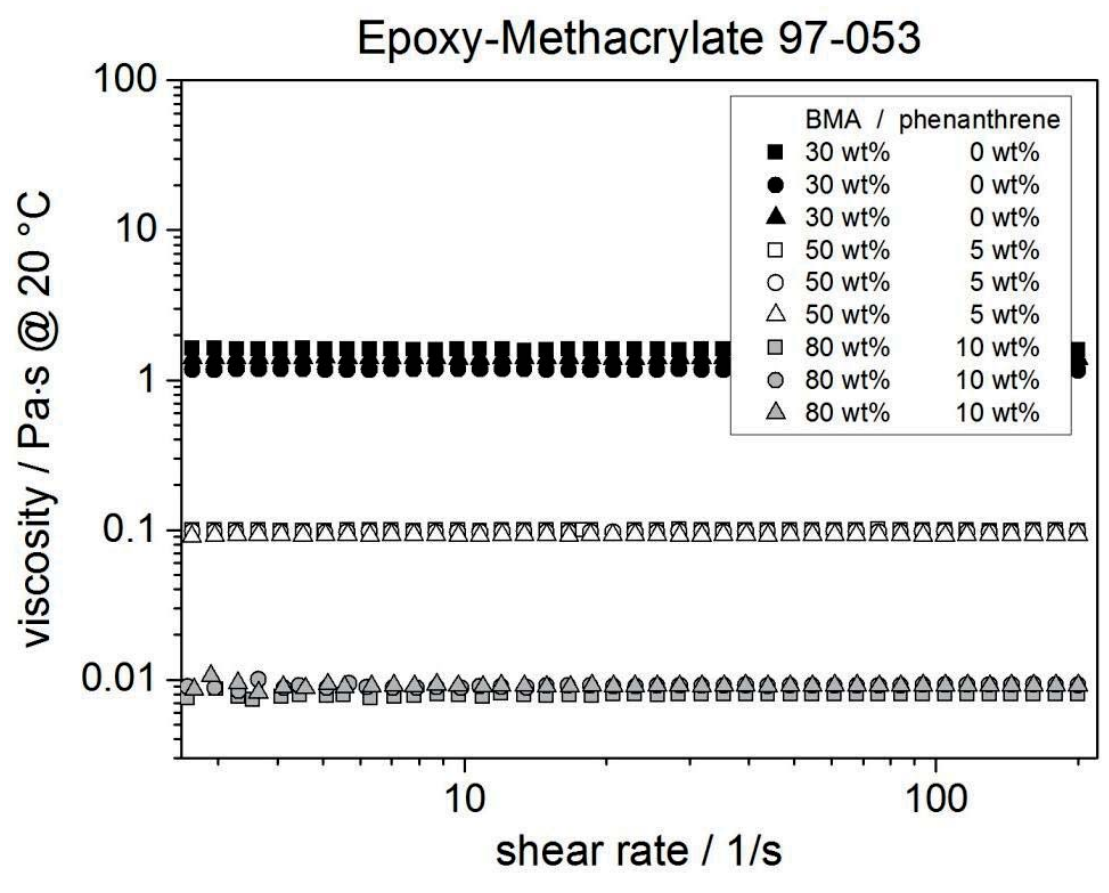




\section{Planar Optronic Systems}

\section{Viscosity of Epoxy Methacrylate 97-053 + BMA}

- Dependence on BMA

- Dependence on phenanthrene
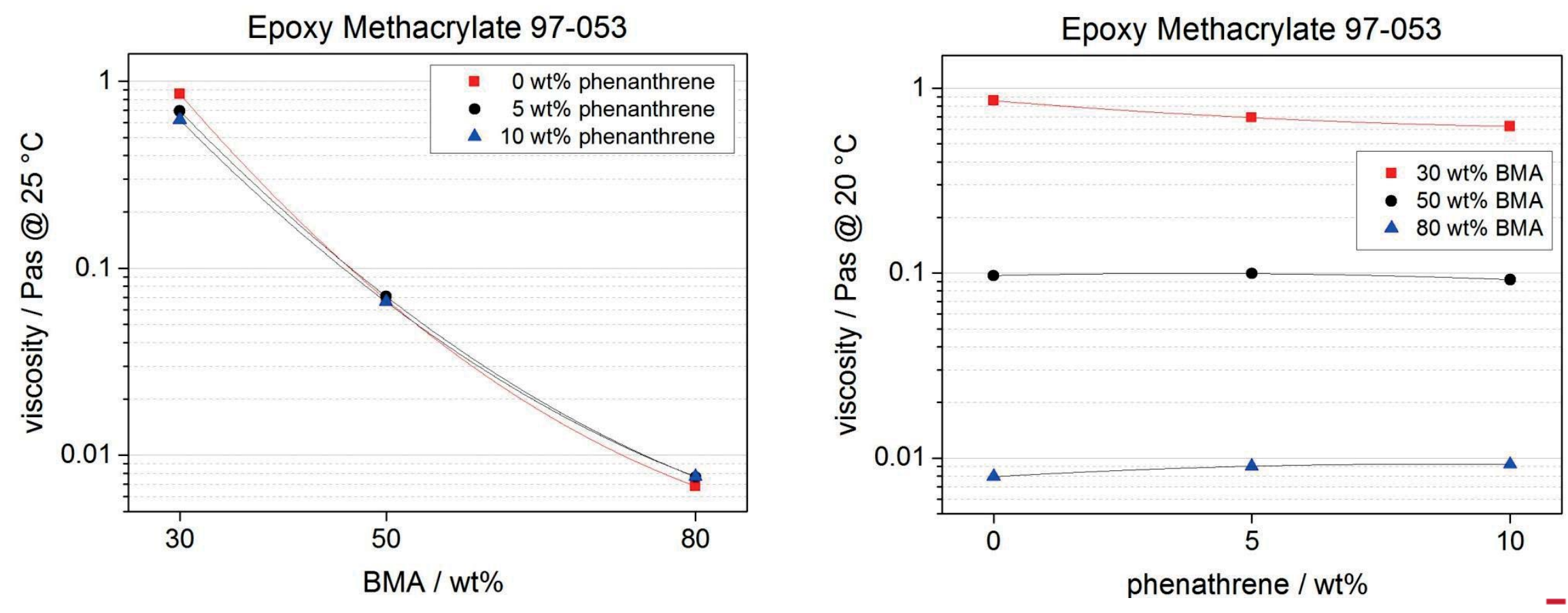


\section{Planar Optronic Systems}

\section{Refractive index}

Polymerized samples

- Phenanthrene increases refractive index

$0 \mathrm{wt} \%: 1.568-1.570$

$5 \mathrm{wt} \%: 1.575-1.577$

$10 \mathrm{wt} \%: 1.585-1.586$

- BMA has low influence

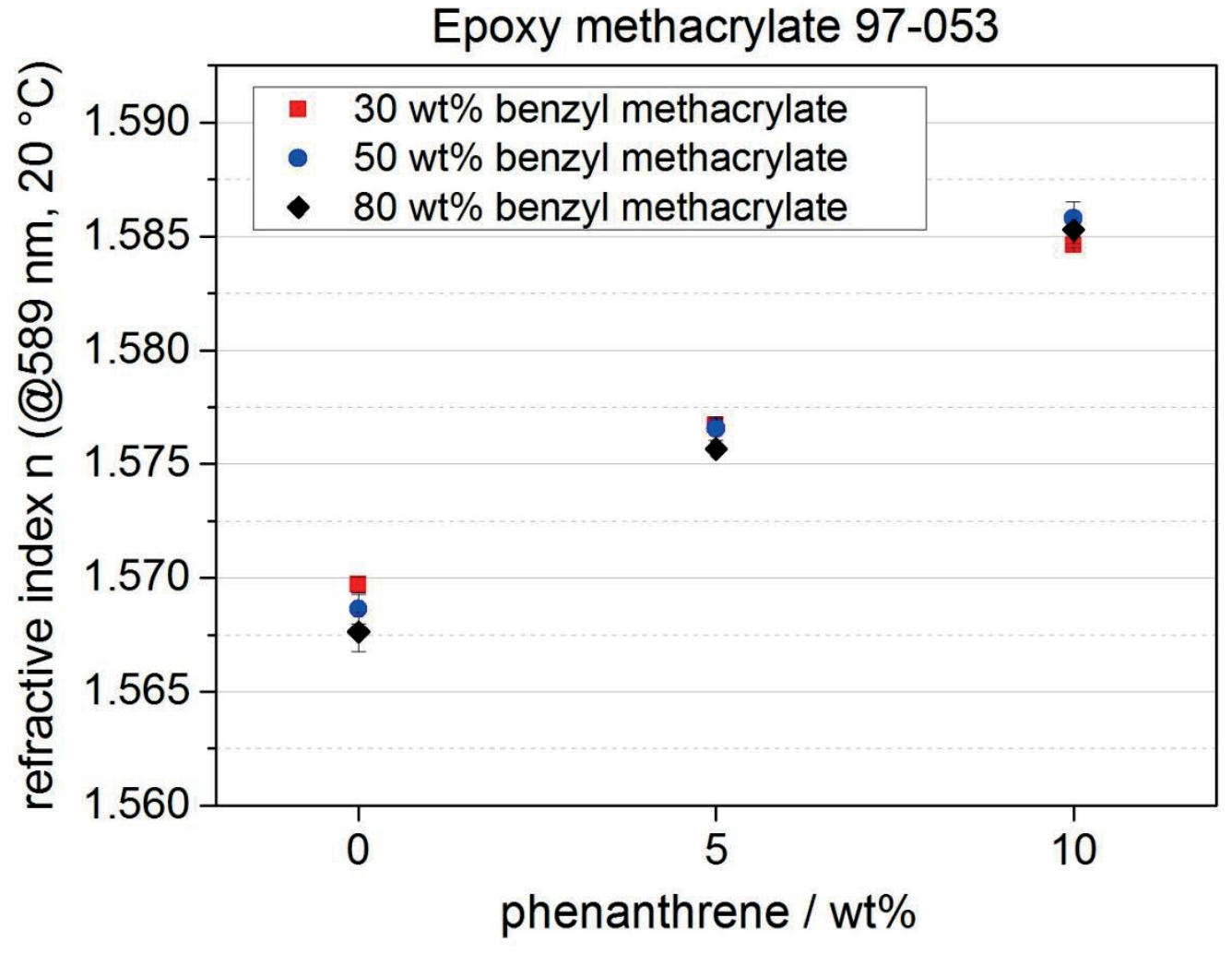

Epoxy methacrylate 97-053

phenanthrene / wt \% 


\section{Planar Optronic Systems}

\section{Data combined}

\section{Viscosity}

$>$ Refractive index

- Refractive index

Viscosity

- Easy lookup

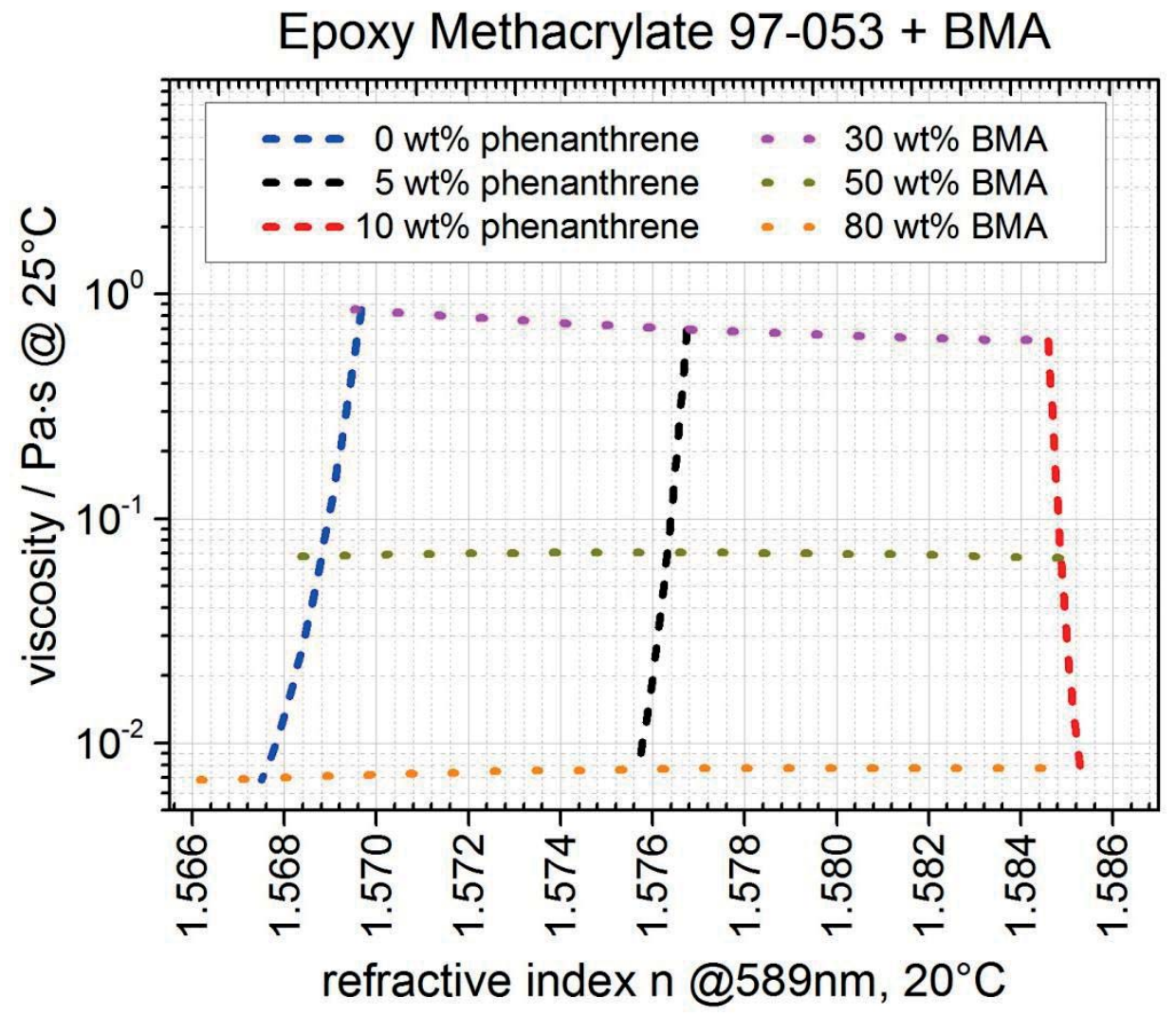




\section{Planar Optronic Systems}

\section{Abbe number}

Polymerized samples

- Phenanthrene decreases Abbe number

- BMA has low influence

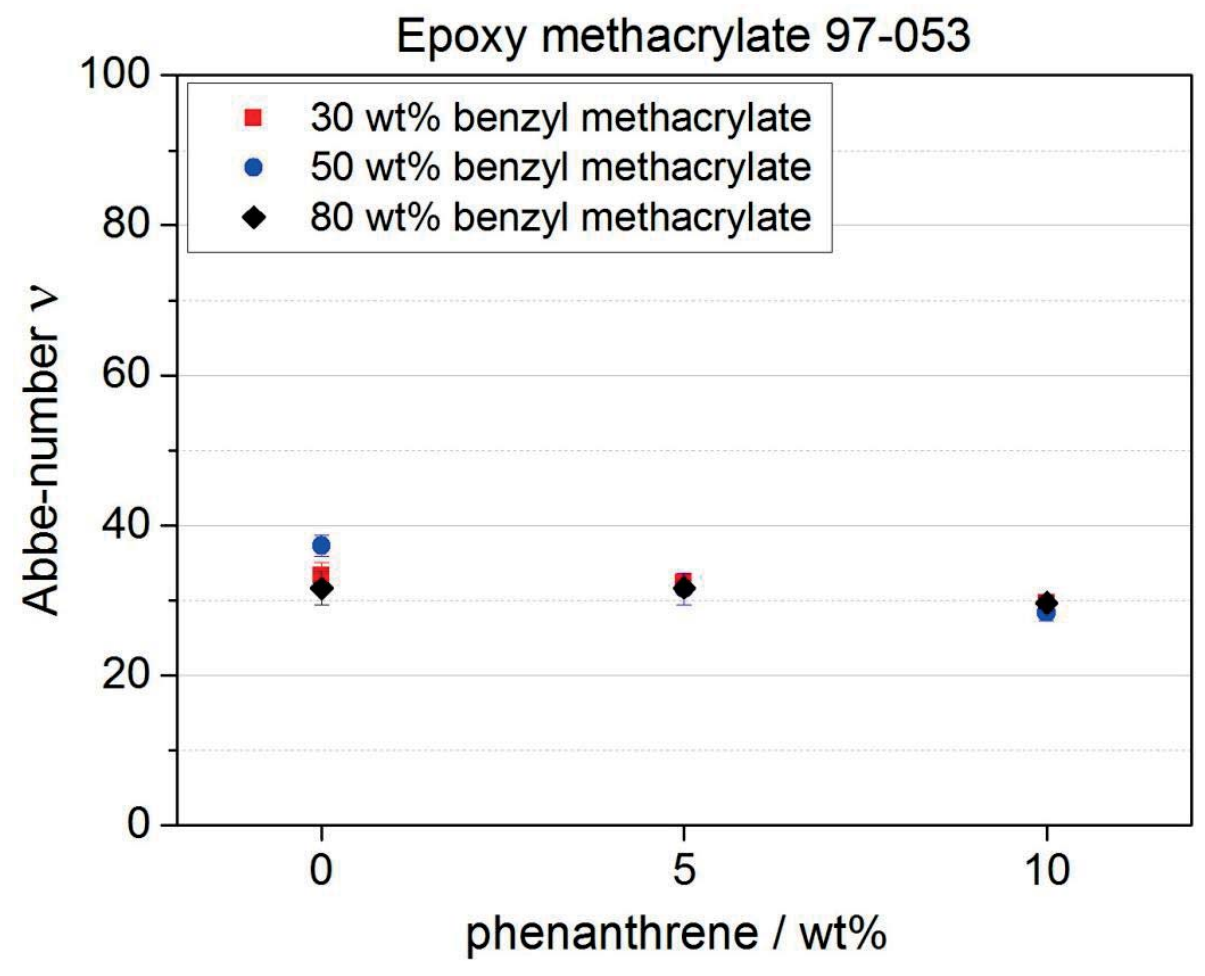




\section{Planar Optronic Systems}

\section{Optical damping of Epoxy Methacrylate (EM) + BMA}

- Independent on BMA

- Dependent on phenanthrene
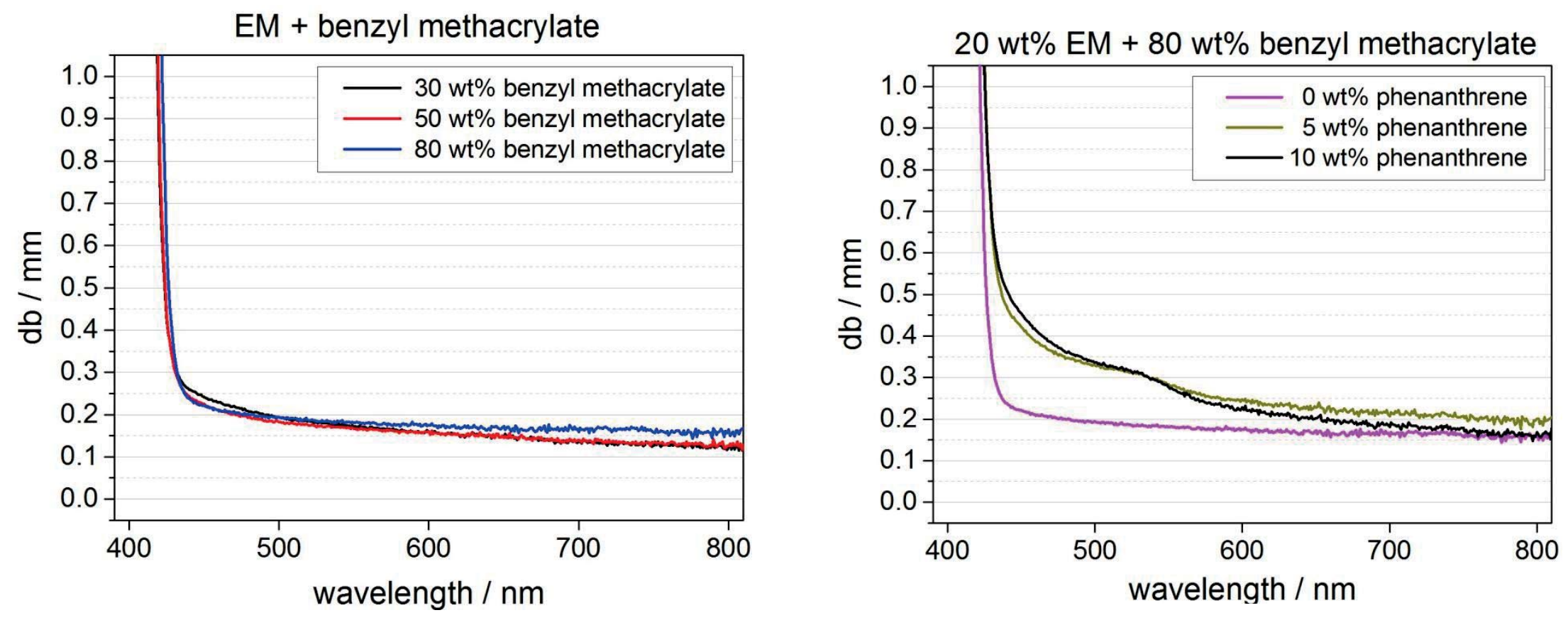


\section{Planar Optronic Systems}

\section{Printed "waveguide"}

IInk-jet

width appr. $180 \mu \mathrm{m}$

height appr. $40 \mu \mathrm{m}$

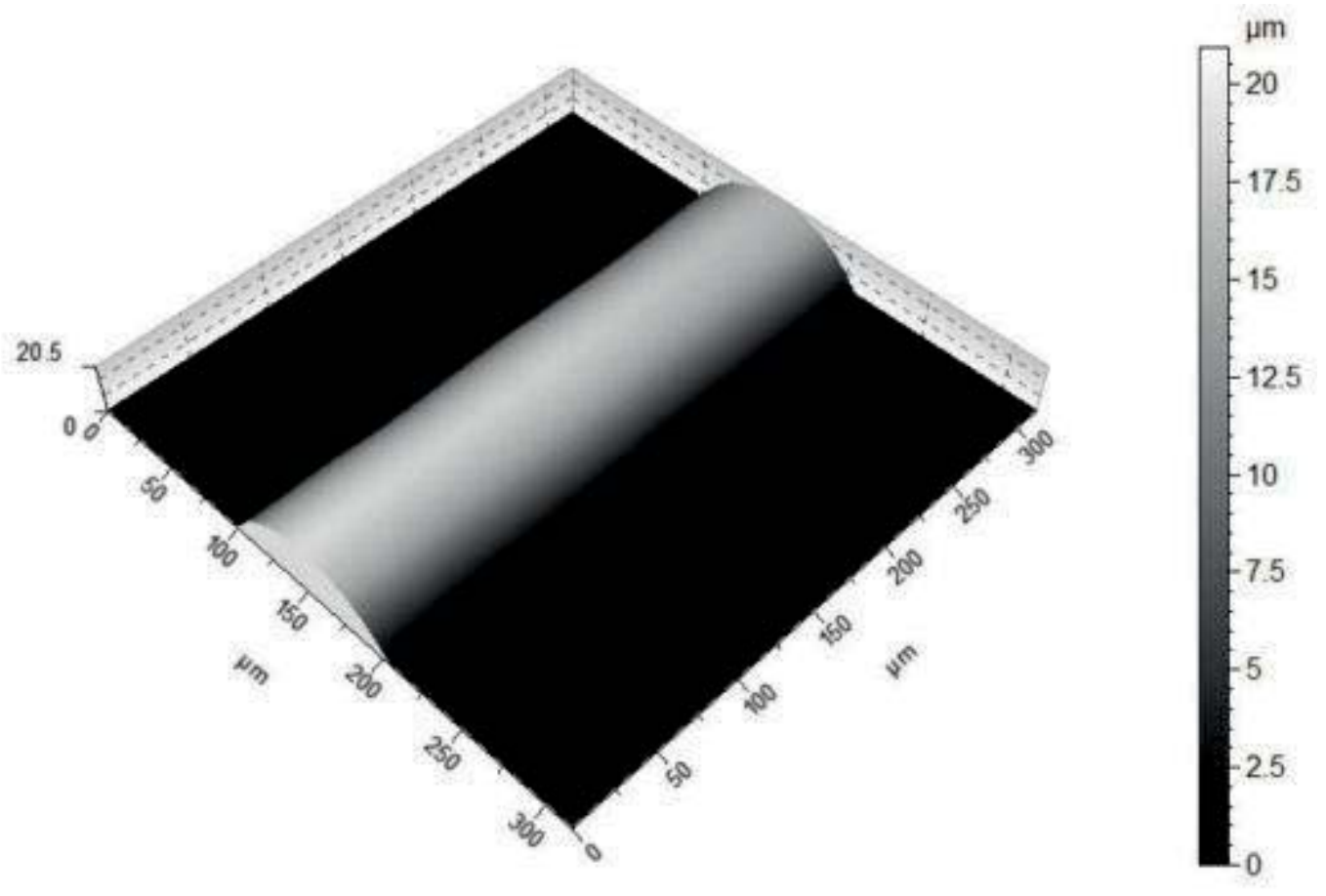

Image of inkjet-printed waveguide taken by confocal microscopy

[Wolfer et al., Procedia Technology, 2014] 


\section{Planar Optronic Systems}

\section{Spincoated waveguide}

- width appr. $10 \mu \mathrm{m}$

- height appr. $5 \mu \mathrm{m}$

- monomode

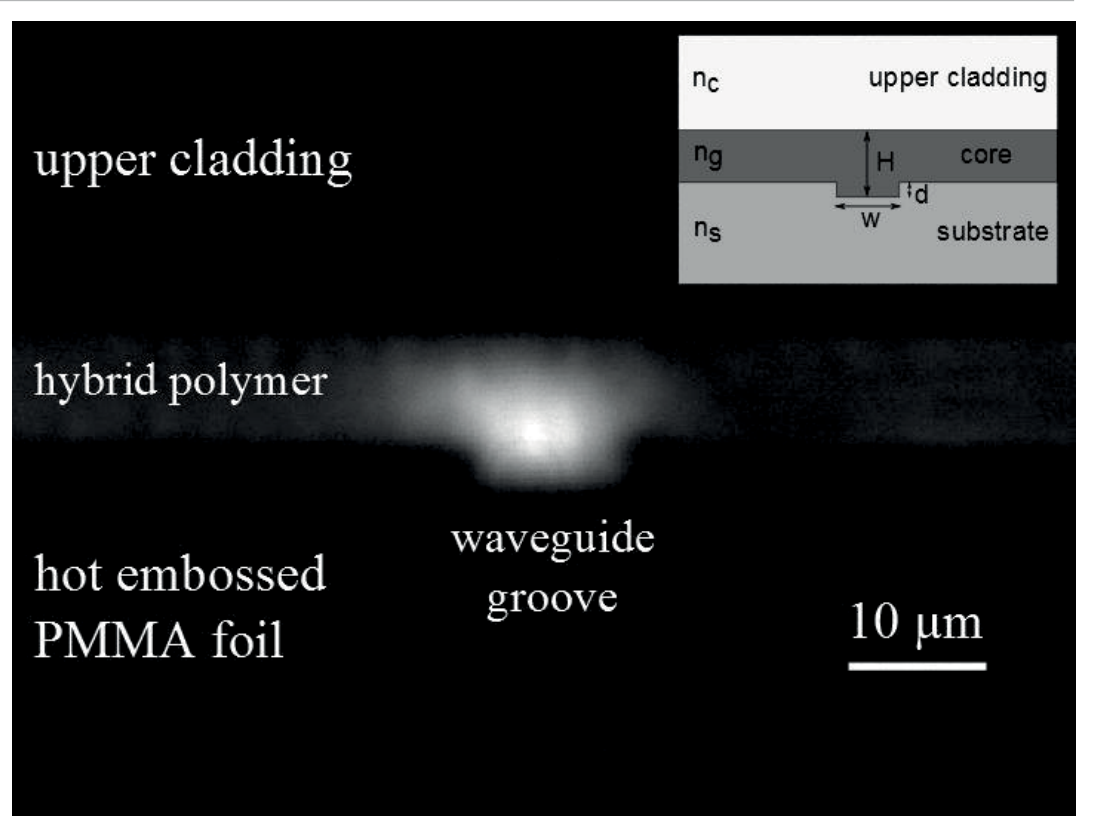

Output facet of a fabricated single-mode inverted rib wageguide [Gleissner et al., Eurosensors Conference, 2015] 


\section{Planar Optronic Systems}

\section{Self writing waveguide}

- Laser writing through monomer

- Between two fibers

- Low loss connection
(1) Butt coupled optical fibers

(2) Polymer applied

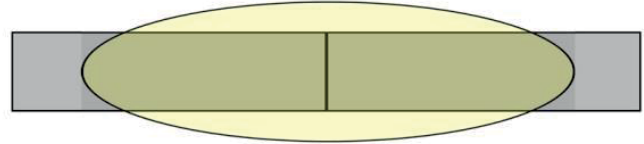

(3) Separation step

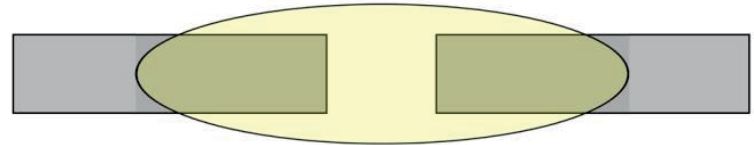

(4) Self-written step upon exposure to $405 \mathrm{~nm}$ laser

(5) Flood UV

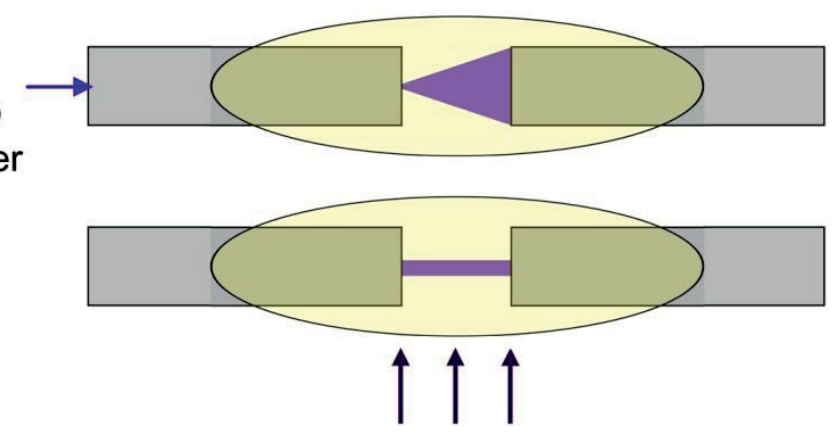

Schematic of the different process steps of the self-written waveguide formation [Günther et al., Optics Letters, 2015] 


\section{Planar Optronic Systems}

\section{Summary}

Viscosity adjustable in a wide range

> $1.5 \mathrm{~Pa} \cdot \mathrm{s}>\eta>8 \mathrm{mPa} \cdot \mathrm{s}\left(@ 20^{\circ} \mathrm{C}\right.$ )

$>$ Suitable for different shaping methods

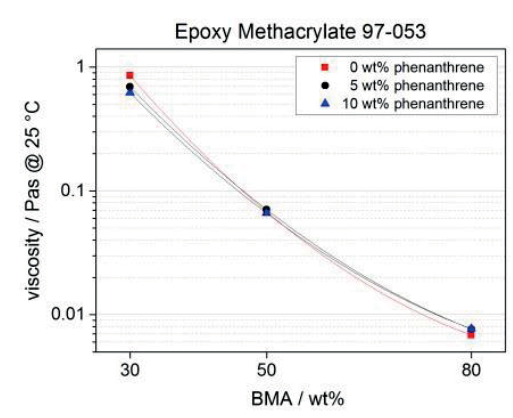

$>$ Range can be extended 


\section{Planar Optronic Systems}

\section{Summary}

- Viscosity adjustable in a wide range

> $1.5 \mathrm{~Pa} \cdot \mathrm{s}>\eta>8 \mathrm{mPa} \cdot \mathrm{s}\left(@ 20^{\circ} \mathrm{C}\right.$ )

$>$ Suitable for different shaping methods

- Range can be extended

- Refractive index tunable

$>1.570<\mathrm{n}<1.585$ (@ $20^{\circ} \mathrm{C}, 589 \mathrm{~nm}$ )

Independent of viscosity
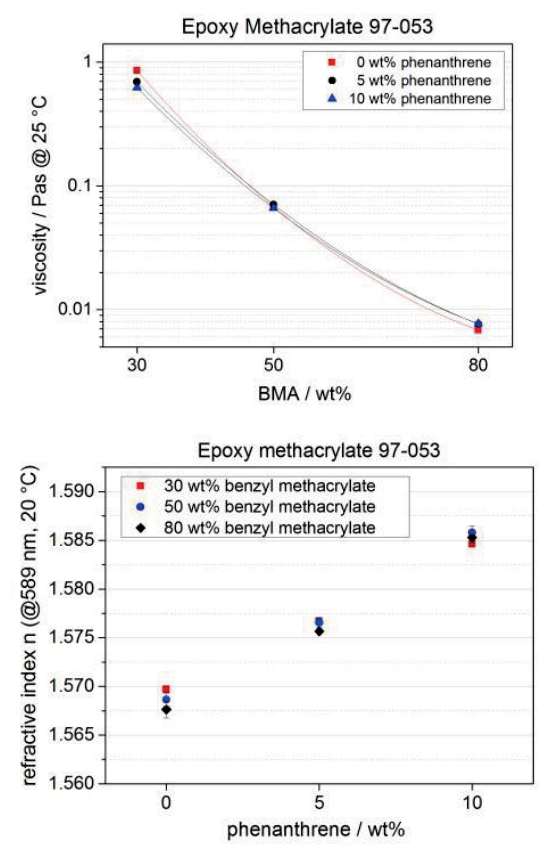


\section{Planar Optronic Systems}

\section{Summary}

- Viscosity adjustable in a wide range

$>1.5 \mathrm{~Pa} \cdot \mathrm{s}>\mathrm{\eta}>8 \mathrm{mPa} \cdot \mathrm{s}\left(@ 20^{\circ} \mathrm{C}\right)$

$>$ Suitable for different shaping methods

$>$ Range can be extended

- Refractive index tunable

$>1.570<\mathrm{n}<1.585$ (@ $20^{\circ} \mathrm{C}, 589 \mathrm{~nm}$ )

$>$ Independent of viscosity
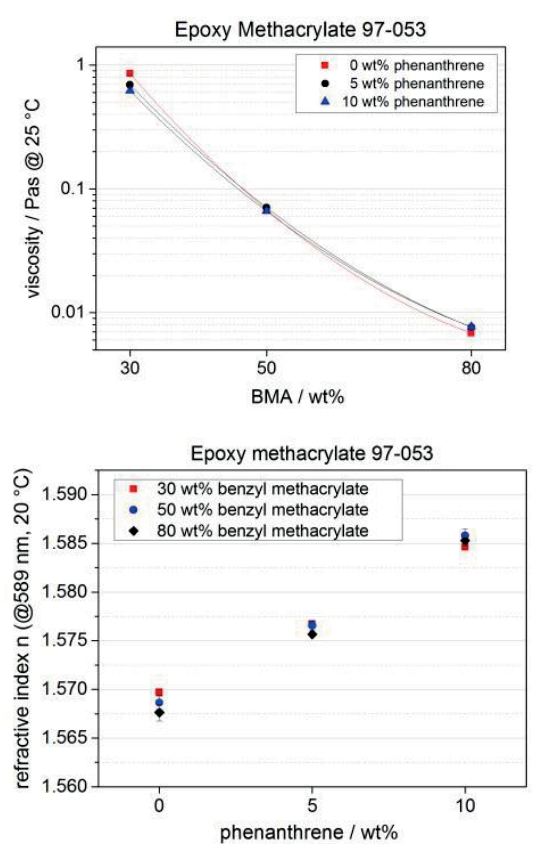

- Dispersion

Abbe numbers: 29 to 38

Optical damping

As low as $0.15 \mathrm{~dB} / \mathrm{mm} @ 600 \mathrm{~nm}$
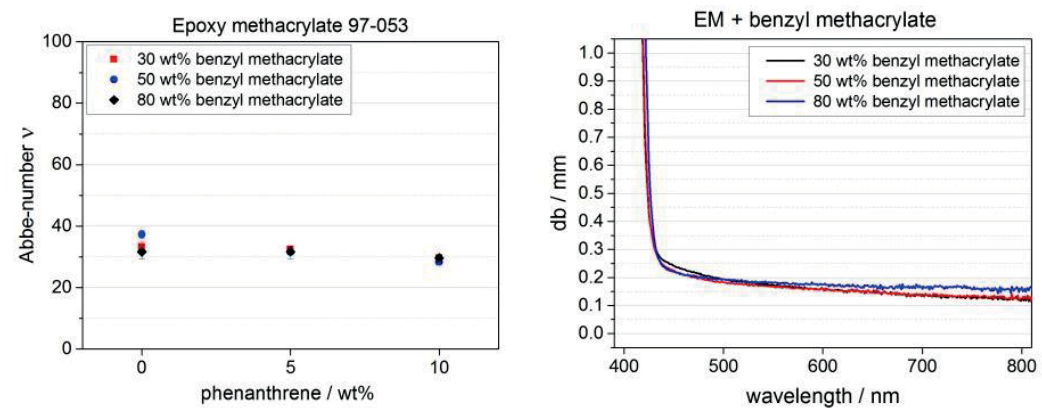


\section{Acknowledgements}

\section{The PlanOS science team (alphabetical order):}

Meriem Akin

Florian Bär

Konrad Bethmann

Tobias Birr

Patrick Bollgrün

Kort Bremer

Boris Chichkov

Ayhan Demircan

Sebastian Dikty

Sebastian Döhring

Henrik Ehlers

Ludmila Eisner

Melanie Gauch

Uwe Gleißner

Axel Günther

\author{
Thomas Hanemann \\ Meike Hofmann \\ Christian Kelb \\ Ann-Katrin Kniggendorf \\ Michael Köhring \\ Martin Körner \\ Jan Gerrit Korvink \\ Wolfgang Kowalsky \\ Dario Mager \\ Uwe Morgner \\ Claas Müller \\ Gregor Osterwinter \\ Torsten Otto \\ Ludger Overmeyer \\ Malwina Pajestka
}

Welm Pätzold

Ann Britt Petermann

Elke Pichler

Oswald Prucker

Torsten Rabe

Maik Rahlves

Holger Reinecke

Carsten Reinhardt

Eduard Reithmeier

Maher Rezem

Lutz Rissing

Detlef Ristau

Bernhard Roth

Raimund Rother

Jürgen Rühe
Laszlo Sajti

Wolfgang Schade

Thomas Schmidt

Anne-Katrin Schuler

Andreas Schwenke

Stanislav Shermann

Yixiao Wang

Nan Wang

Ulrike Willer

Tim Wolfer

Merve Wollweber

Marc Wurz

Yanfen Xiao

Hans Zappe

Urs Zywietz

Funded by German Research Foundation

(Deutsche Forschungsgemeinschaft)

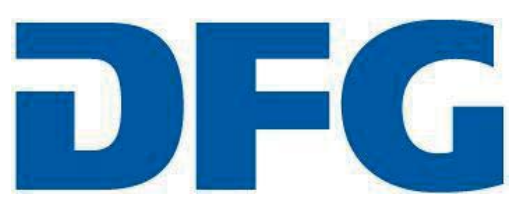

